Article

\title{
The Supportive Care Needs of Regional and Remote Cancer Caregivers
}

\author{
Anna Stiller $1,2, * \mathbb{C}$, Belinda C. Goodwin 1,2(D), Fiona Crawford-Williams $1,2,3(\mathbb{D}$, Sonja March 2,4 , \\ Michael Ireland ${ }^{2,4} \mathbb{D}^{\text {, Joanne F. Aitken }}{ }^{1,2,5,6}$, Jeff Dunn ${ }^{1,2,7,8,9}$ and Suzanne K. Chambers $9,10,11$
}

1 Viertel Cancer Research Centre, Cancer Council Queensland, Fortitude Valley, Brisbane, QLD 4006, Australia; belindagoodwin@cancerqld.org.au (B.C.G.); fiona.crawfordwilliams@qut.edu.au (F.C.-W.); joanneaitken@cancerqld.org.au (J.F.A.); jeff.dunn@pcfa.org.au (J.D.)

2 Centre for Health Research, University of Southern Queensland, Springfield, QLD 4300, Australia; sonja.march@usq.edu.au (S.M.); michael.ireland@usq.edu.au (M.I.)

3 Cancer Palliative Care Outcomes Centre, Queensland University of Technology, Kelvin Grove, QLD 4059, Australia

4 School of Psychology and Counselling, University of Southern Queensland, Ipswich, QLD 4305, Australia

5 School of Public Health and Social Work, Queensland University of Technology, Kelvin Grove, QLD 4059, Australia

6 School of Public Health, The University of Queensland, Springfield, QLD 4300, Australia

7 Menzies Health Institute Queensland, Griffith University, Mt Gravatt, QLD 4222, Australia

8 Prostate Cancer Foundation of Australia, St Leonards, Sydney, NSW 2065, Australia

9 Faculty of Health, University of Technology Sydney, Ultimo, Sydney, NSW 2007, Australia; suzanne.chambers@acu.edu.au

10 Faculty of Health Sciences, Australian Catholic University, Banyo, Brisbane, QLD 4014, Australia

11 Exercise Medicine Research Institute, Edith Cowan University, Joondalup, Perth, WA 6027, Australia

* Correspondence: annastiller@cancerqld.org.au

check for

updates

Citation: Stiller, A.; Goodwin, B.C.; Crawford-Williams, F.; March, S.; Ireland, M.; Aitken, J.F.; Dunn, J.; Chambers, S.K. The Supportive Care Needs of Regional and Remote Cancer Caregivers. Curr. Oncol. 2021, 28, 3041-3057. https://doi.org/ 10.3390 /curroncol28040266

Received: 13 July 2021

Accepted: 3 August 2021

Published: 9 August 2021

Publisher's Note: MDPI stays neutral with regard to jurisdictional claims in published maps and institutional affiliations.

Copyright: (c) 2021 by the authors. Licensee MDPI, Basel, Switzerland This article is an open access article distributed under the terms and conditions of the Creative Commons Attribution (CC BY) license (https:// creativecommons.org/licenses/by/ $4.0 /)$.
Abstract: Objective: As cancer survival rates continue to increase, so will the demand for care from family and friends, particularly in more isolated settings. This study aims to examine the needs of cancer caregivers in regional and remote Australia. Methods: A total of 239 informal (i.e., non-professional) cancer caregivers (e.g., family/friends) from regional and remote Queensland, Australia, completed the Comprehensive Needs Assessment Tool for Cancer Caregivers (CNAT-C). The frequencies of individuals reporting specific needs were calculated. Logistic regression analyses assessed the association between unmet needs and demographic characteristics and cancer type. Results: The most frequently endorsed needs were lodging near hospital (77\%), information about the disease $(74 \%)$, and tests and treatment $(74 \%)$. The most frequent unmet needs were treatment near home (37\%), help with economic burden (32\%), and concerns about the person being cared for $(32 \%)$. Younger and female caregivers were significantly more likely to report unmet needs overall $(\mathrm{OR}=2.12 ; \mathrm{OR}=0.58)$, and unmet healthcare staff needs $(\mathrm{OR}=0.35 ; \mathrm{OR}=1.99$, respectively). Unmet family and social support needs were also significantly more likely among younger caregivers $(\mathrm{OR}=0.35)$. Caregivers of breast cancer patients $(\mathrm{OR}=0.43)$ and older caregivers $(\mathrm{OR}=0.53)$ were significantly less likely to report unmet health and psychology needs. Proportions of participants reporting needs were largely similar across demographic groups and cancer type with some exceptions. Conclusions: Caregiver health, practical issues associated with travel, and emotional strain are all areas where regional and remote caregivers require more support. Caregivers' age and gender, time since diagnosis and patient cancer type should be considered when determining the most appropriate supportive care.

Keywords: cancer; caregivers; supportive care needs; rural; regional and remote

\section{Introduction}

Cancer, as a disease group, is the leading cause of disease burden in Australia and the economic cost of cancer to the health system is substantial [1,2]. Caregivers play an indispensable role in maintaining the livelihood and well-being of cancer patients. A caregiver 
is defined here as the person (e.g., partner, family member or friend) "nominated by the cancer patient as most involved in supporting them through the illness" [3]. Caregivers help the patient in a myriad of ways such as in managing symptoms and side-effects, providing assistance at medical appointments, giving emotional support, assisting in activities of daily living, as well as taking on extra family and household responsibilities [4].

Caring for someone with cancer, particularly a loved-one, can incur significant physical and emotional burdens for the caregiver [5-7]. While concurrently dealing with their own emotional and existential concerns, caregiving demands may at times exceed the caregiver's capabilities and resources [8]. As cancer survival rates continue to increase, so will the demand for care and support from family and friends. Therefore, it is vital that caregivers needs are assessed and addressed in order to support their own well-being and maximize their capacity to provide care for the patient [9].

Not all needs are of equal importance to all caregivers [10]. Age (being younger) and gender (being female) have been associated with a higher frequency of unmet needs among caregivers [3,9-12]. Kim and colleagues [10] found that younger caregivers were more likely to report unmet psychosocial and financial needs at two years post-diagnosis, and unmet needs for managing loss if they were bereaved caregivers at five years. The patient's cancer type is also associated with caregiver needs. For example, research has found that caregivers of patients with haematological cancer have more unmet needs compared to caregivers of patients with solid tumours [13]; and caregivers of those with lung cancer and non-Hodgkins lymphoma have a higher frequency of health care service needs and information needs, respectively, compared to other cancer types [3].

There are unique burdens and challenges for caregivers living in regional and remote areas [14-16]. They may experience poorer access to health care and support services, difficulties coping with the logistical challenges of regular travel or relocation during the patient's treatment, disruption to work capacity, financial instability, and social disconnection [14-16]. Furthermore, in regional and remote communities where access to medical facilities is limited, caregivers may be required to take on roles that in urban areas would be fulfilled by professional support services. Rural and remote areas typically lack the infrastructure, specialist and allied health services found in more densely populated urban areas. As a result the increase in early discharge procedures in the healthcare system may have a considerable impact on caregivers in rural and remote areas, who are taking up the majority of the day-to-day responsibility during the patient recovery and rehabilitation period $[17,18]$. Consequently, the magnitude of caregiver needs may also vary depending on geographical location. For instance, among a sample of caregivers of people with haematological cancers, those living in rural areas had a higher prevalence of financerelated unmet needs compared to those from urban areas (e.g., unmet need in 'finding information about financial help'-rural caregivers $20 \%$ vs. urban $13 \%$ [15]). Therefore, it is essential to establish the importance of varying needs in this group and investigate any difference according to demographic, geographical, and disease characteristics. In order to tailor appropriate support and information resources to cancer caregivers from regional or remote areas, identifying and addressing the support needs of cancer caregivers in regional and remote communities is particularly important and a vital element in improving health and well-being outcomes in these areas.

The aim of this study is to describe the types and prevalence of supportive care needs of cancer caregivers in regional and remote Australia, both in terms of prevalence of needs and degree of unmet need and to compare unmet need according to the caregiver's age group, gender, geographical location, socio-economic status, the length of time since diagnosis, and the cancer type of the patient. This information will assist in identifying areas where support is most needed for cancer caregivers living in regional and remote communities and may help to inform future supportive care interventions. 


\section{Materials and Methods}

\subsection{Participants}

Participants were the nominated caregivers of cancer patients recruited to a larger research project. The cancer patients were staying at subsidised accommodation lodges while receiving their cancer care in a major centre more than $50 \mathrm{~km}$ from their home. Details of patient recruitment is detailed elsewhere [19]. Caregivers included spouses/partners, family members, or friends. The eligibility criteria included being over 18 and being able to read and understand English. Caregivers did not have to accompany the cancer patient to the accommodation lodge, and did not have to be living in the same personal residence as the cancer patient, in order to be recruited to the study. They were not professional paid caregivers; however, it is possible that some may have been claiming a carer's payment from the government (this information was not collected).

\subsection{Recruitment}

Cancer patients participating in the larger study nominated 402 caregivers and provided their contact details. The research team contacted nominated caregivers via telephone, inviting them to participate and sending out an invitation pack, which included consent forms and a questionnaire, if they were interested in participating. Of these, 259 consented to participate and 239 caregivers provided complete data for the current study. Figure 1 depicts the participant recruitment flow chart.

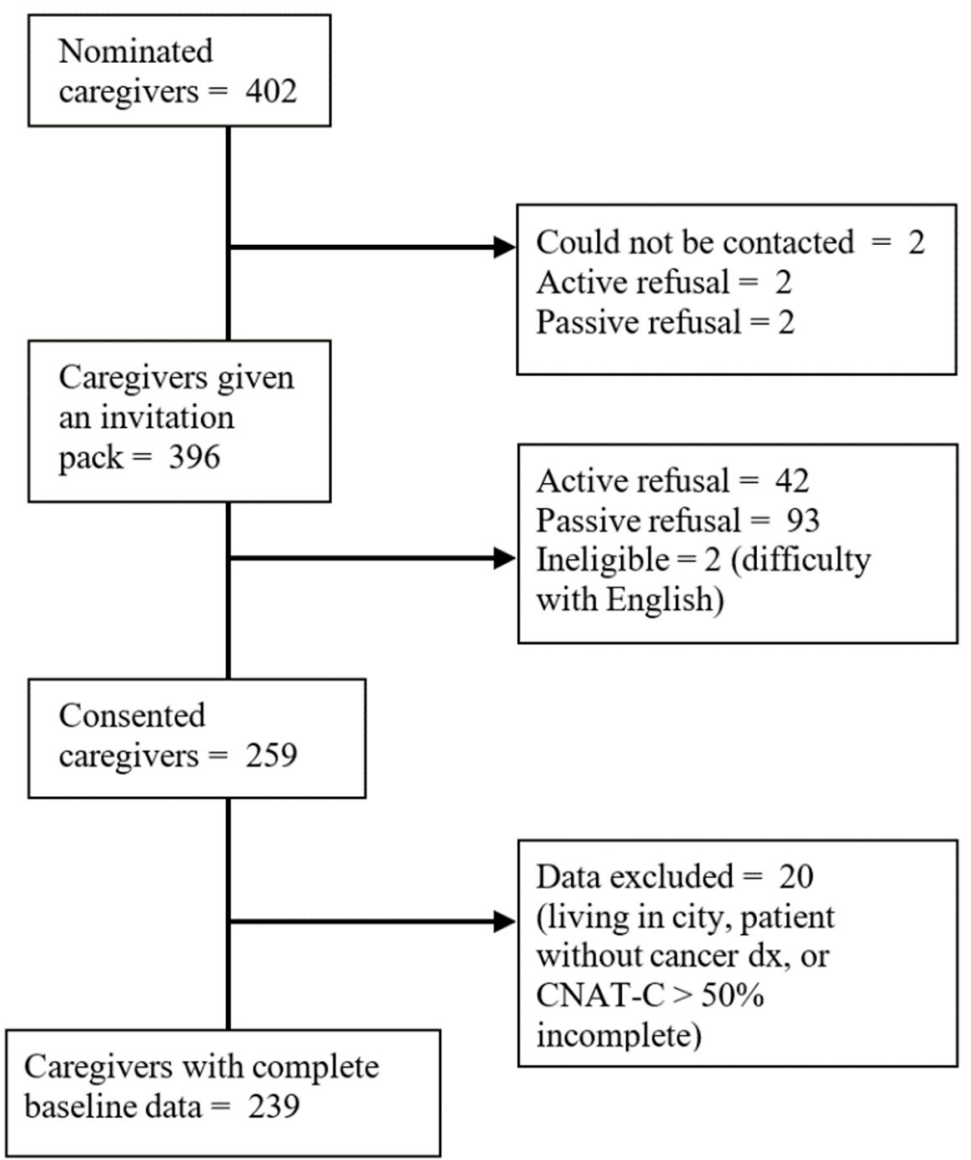

Figure 1. Recruitment flowchart.

\subsection{Materials}

Assessments included a self-administered questionnaire (SAQ) and face-to-face or telephone interview at baseline. These instruments captured data such as patient and caregivers' experiences, psycho-social wellness, and satisfaction with their health care, 
along with the demographic and caregiver supportive care needs measure used in the current study.

\subsection{Measures}

\subsubsection{Caregivers' Demographic Characteristics}

Gender, age, country of birth, and highest level of education were reported by each participant. Their residential street address at baseline was geocoded and mapped to the 2011 SA2 boundaries using MapMarker Australia V.15.16.0.21 and MapInfo Pro V.5.0 and classified by Remoteness Area [20] and Socioeconomic Index for Areas (SEIFA) [21].

\subsubsection{Patient Diagnosis Information}

The most recently diagnosed primary cancer site of the patient for whom they were caring was obtained via self-report from the patient and verified in the population-based Queensland Cancer Register (QCR). Patient self-report data were relied on where diagnosis could not be verified by the QCR $(n=22)$, for example, if the patient had non-melanoma skin cancer (which is not routinely notified to registries in Australia) or the patient's diagnosis was very recent and had not yet been notified to the QCR.

\subsubsection{Caregiver Supportive Care Needs}

There are several useful measures of cancer caregiver need that have been utilised and validated in Australian samples [8,22,23]. However, given the unique challenges for caregivers in regional and remote areas, the Comprehensive Needs Assessment Tool for Cancer Caregivers (CNAT-C) [24] was used in the current study due to the inclusion of a 'practical support' domain that covers needs such as treatment near home and transport service, which may be particularly relevant for those living in more remote, underserviced areas. The CNAT-C is a comprehensive needs assessment tool for caregivers of cancer patients, that includes 41 items across 7 domains: health and psychological problems (e.g., need for help with 'my own health problems'); family/social support (e.g., 'help with difficulties in family relationships after cancer diagnosis'); health-care staff (e.g., 'being respected and treated as a person by my doctor'); information (e.g., 'information about tests and treatment'); religious/spiritual support (e.g., 'help in finding the meaning of my situation and coming to terms with it'); hospital facilities and services (e.g., 'need for space reserved for caregivers'); and practical support (e.g., 'lodging near hospital'). Items were answered on a 5-point Likert scale: "no need/not applicable"; "no need/satisfied"; "low need"; "moderate need"; and "high need", referring to the past month. The original CNAT-C uses a 4-point scale, however, in order to distinguish those with no need and those who had no need because the need was satisfied, an extra response item was added. (i.e., need satisfied).

Given the CNAT-C has not been validated for use outside of Korea and China [24,25], a confirmatory factor analysis (CFA) was conducted to assess whether the proposed domains were applicable in a regional and remote Australian setting. The CFA, conducted in Mplus v8, used a weighted least-squares mean and variance adjusted (WLMSV) estimator [26]. The CFA demonstrated reasonable fit for the original scale in this sample $\left(\chi^{2}=1545.88\right.$, $p<0.001, \mathrm{RMSEA}=0.066, p<0.001, \mathrm{CFI}=0.915, \mathrm{TLI}=0.908)$. All but one item loaded above 0.65 on their respective factors (Tables A1 and A2). The religious/spiritual support factor contained only two items and the item 'religious support' had a factor loading of 0.12 while the second item 'help in finding the meaning of my situation and coming to terms with it' had a factor loading of 3.69. Modification indices suggested substantial improvements in model fit could be achieved by removing both of these items, which also demonstrated poor variance and insufficient endorsement in this sample (i.e., 1.67\% and $7.11 \%$ non-zero responses, respectively). A second model was assessed excluding the religious/spiritual support factor (and the items within). The second model demonstrated better fit $\left(\chi^{2}=1090.81, p<0.001, \mathrm{RMSEA}=0.050, p=0.542, \mathrm{CFI}=0.955, \mathrm{TLI}=0.951\right)$ and 
the factor structure remained stable with all factor loadings above 0.65 . Religiosity items were therefore not included in the main analysis in this study.

Multiple scoring approaches were applied to create three versions of the subscale scores. These included a variable reflecting 'need' (whether met or unmet), which was calculated whereby $0=$ not applicable; and $1=$ satisfied, low need, moderate need, or high need. A second variable reflecting 'Unmet need' was calculated where $0=$ not applicable, or satisfied; and $1=$ low, moderate, or high need and a third reflecting 'Degree of unmet need' was also calculated for each item where $0=$ not applicable or satisfied; $1=$ low need; 2 = moderate need; and $3=$ high need.

\subsection{Data Analysis}

Frequencies and proportions of need (i.e., prevalence of need regardless of being met or unmet) and unmet need for each item, and unmet need for each domain, were calculated. Comparisons of prevalence of unmet need in each domain were made across age, gender, area level and cancer type groupings using logistic regression. In models where age of caregiver was an independent variable, age of patient was controlled for. Where $p$ values less than 0.05 indicated significant group differences existed for variables with more than two categories (i.e., age and time since diagnosis), polynomial contrasts were assessed. In addition, frequencies of unmet need for single items were compared through ranking the percentage of participants reporting at least some need for each item across age, gender, area level and cancer type group; a descriptive method applied in previous similar studies for identifying levels of need across groups [5,15,27].

\section{Results}

\subsection{Sample Characteristics}

Most caregivers in the sample were the spouse or partner of the person with cancer $(83 \%)$, female (62\%) and born in Australia (81\%). The mean age of caregivers was 62.0 years (standard deviation $(\mathrm{SD})=12.6$, range 18-91). The median time since the patient's diagnosis was 8.9 months (interquartile range: 4.8 to 23.3). Ninety-five percent of the sample were within 84 months ( 7 years) from the patient's diagnosis, with one patient being diagnosed with their current primary cancer 25 years ago. Caregivers were mostly caring for patients who had been diagnosed with breast $(18 \%)$, skin $(14 \%)$, prostate $(12 \%)$ or head and neck $(11 \%)$ cancer. Most resided in inner (52\%) and outer $(43 \%)$ regional areas, with only $5 \%$ residing in remote or very remote areas. Caregivers resided in predominantly low socio-economic areas. See Table 1 for further details of the demographic characteristics of caregivers in this sample.

Table 1. Sample characteristics.

\begin{tabular}{ccc}
\hline & $\boldsymbol{n}^{\mathbf{1}}$ & $\%$ \\
\hline Gender & & \\
\hline Female & 146 & $61.6 \%$ \\
Male & 91 & $38.4 \%$ \\
\hline Relationship to patient & & \\
\hline Spouse/Partner & 164 & $83.3 \%$ \\
Other relative & 23 & $11.7 \%$ \\
Other non-relative & 10 & \\
\hline Highest level of education & & $42.1 \%$ \\
\hline Year 10 or below & 95 & $13.4 \%$ \\
Senior high school & 30 & $44.2 \%$ \\
Tertiary (Tafe/Uni) & 99 & \\
\hline ATSI & & $98.3 \%$ \\
\hline No & 224 & $1.8 \%$ \\
\hline Yes & 4 &
\end{tabular}


Table 1. Cont.

\begin{tabular}{|c|c|c|}
\hline & $n^{1}$ & $\%$ \\
\hline \multicolumn{3}{|l|}{ Country of birth } \\
\hline Australia & 165 & $81.3 \%$ \\
\hline United Kingdom & 17 & $8.4 \%$ \\
\hline New Zealand & 10 & $4.9 \%$ \\
\hline Other & 11 & $5.4 \%$ \\
\hline \multicolumn{3}{|l|}{ Area-level disadvantage (SEIFA) } \\
\hline 1st Quintile (lowest) & 94 & $39.5 \%$ \\
\hline 2nd & 74 & $31.1 \%$ \\
\hline $3 \mathrm{rd}$ & 45 & $18.9 \%$ \\
\hline 4th & 23 & $9.7 \%$ \\
\hline 5th Quintile (highest) & 2 & $0.8 \%$ \\
\hline \multicolumn{3}{|l|}{ Remoteness } \\
\hline Inner regional & 124 & $52.3 \%$ \\
\hline Outer regional & 101 & $42.6 \%$ \\
\hline Remote and very remote & 12 & $5.1 \%$ \\
\hline \multicolumn{3}{|l|}{ Cancer type of patient } \\
\hline Breast & 44 & $18.4 \%$ \\
\hline Skin & 34 & $14.2 \%$ \\
\hline Prostate & 28 & $11.7 \%$ \\
\hline Head and neck & 25 & $10.5 \%$ \\
\hline Gynaecological & 21 & $8.8 \%$ \\
\hline Colorectal & 16 & $6.7 \%$ \\
\hline Lung & 11 & $4.6 \%$ \\
\hline Non-Hodgkins lymphoma & 13 & $5.4 \%$ \\
\hline Brain & 5 & $2.1 \%$ \\
\hline Other & 34 & $14.2 \%$ \\
\hline Unknown & 8 & $3.3 \%$ \\
\hline
\end{tabular}

Abbreviations: ATSI $=$ Aboriginal or Torres Strait Islander; Tafe $=$ Technical and further education institution Uni $=$ University. ${ }^{1}$ Total $n$ does not always equal 239 where data was missing.

\subsection{Overall Caregiver Needs}

Overall, $96.2 \%$ of caregivers reported that a need existed for them (regardless of whether it had been met) in at least one domain over the past month, with $71.6 \%$ reporting at least one unmet need over the past month. The majority of caregivers reported that at least one need existed for them in the practical support (86.6\%), information (86.2\%), health-care staff $(82.4 \%)$ (e.g., 'seeing doctor quickly and easily when in need'), health and psychology (80.8\%), and hospital facilities and services (74.5\%) domains (see Table 2). Unmet need was most frequently reported in the practical support $(50.2 \%)$, health and psychological (48.5\%), and information domains $(45.6 \%)$, whereas healthcare staff $(30.5 \%)$, family/social support (32.6\%), and hospital facilities and services (35.2\%) were domains where need was less often reported as unmet (see Table 3).

Table 2. Frequency of responses to CNAT-C items.

\begin{tabular}{|c|c|c|c|c|}
\hline Domain & CNAT-C Item (Ordered by Item Number in Scale) & $\begin{array}{c}\text { Need (Including Met) } \\
\text { N (\%) }\end{array}$ & $\begin{array}{c}\text { Unmet Need } \\
\text { N (\%) }\end{array}$ & $\begin{array}{c}\text { Degree of Need } \\
\text { Mean (SD) }\end{array}$ \\
\hline Health and Psych & $\begin{array}{c}\text { My own health problems } \\
\text { Concerns about the person I provide care for } \\
\text { Depression } \\
\text { Feelings of anger, irritability, or nervousness } \\
\text { Loneliness or feelings of isolation } \\
\text { Feelings of vague anxiety }\end{array}$ & $\begin{array}{c}193(80.8 \%) \\
116(49.2 \%) \\
168(71.2 \%) \\
88(37.5 \%) \\
102(43.4 \%) \\
83(35.2 \%) \\
120(50.9 \%)\end{array}$ & $\begin{array}{l}116(48.5 \%) \\
66(28.0 \%) \\
75(31.8 \%) \\
43(18.3 \%) \\
47(20.0 \%) \\
43(18.2 \%) \\
56(23.7 \%)\end{array}$ & $\begin{array}{l}0.36(0.59) \\
0.43(0.79) \\
0.54(0.91) \\
0.28(0.67) \\
0.30(0.68) \\
0.28(0.68) \\
0.35(0.71)\end{array}$ \\
\hline
\end{tabular}


Table 2. Cont.

\begin{tabular}{|c|c|c|c|c|}
\hline Domain & CNAT-C Item (Ordered by Item Number in Scale) & $\begin{array}{l}\text { Need (Including Met) } \\
\text { N (\%) }\end{array}$ & $\begin{array}{l}\text { Unmet Need } \\
\text { N (\%) }\end{array}$ & $\begin{array}{l}\text { Degree of Need } \\
\text { Mean (SD) }\end{array}$ \\
\hline \multirow[t]{6}{*}{ Family/Social } & \multirow[b]{2}{*}{$\begin{array}{c}\text { Help with over-dependence from the person I am } \\
\text { caring for }\end{array}$} & $151(63.2 \%)$ & $78(32.6 \%)$ & $0.25(0.50)$ \\
\hline & & $82(34.5 \%)$ & $26(10.9 \%)$ & $0.18(0.55)$ \\
\hline & $\begin{array}{c}\text { Help with lack of appreciation of my caregiving from } \\
\text { the person ... }\end{array}$ & $86(36.3 \%)$ & $33(13.9 \%)$ & $0.23(0.64)$ \\
\hline & $\begin{array}{l}\text { Help with difficulties in family relationships after } \\
\text { cancer diagnosis }\end{array}$ & $106(44.4 \%)$ & $37(15.5 \%)$ & $0.24(0.62)$ \\
\hline & $\begin{array}{c}\text { Help with difficulties in interpersonal relationships } \\
\text { after cancer... }\end{array}$ & $106(44.4 \%)$ & $36(15.1 \%)$ & $0.21(0.57)$ \\
\hline & Help with my own relaxation and my personal life & $126(52.7 \%)$ & $54(22.6 \%)$ & $0.37(0.77)$ \\
\hline \multirow[t]{9}{*}{ Healthcare staff } & \multirow{5}{*}{$\begin{array}{c}\text { Being respected and treated as a person by my doctor } \\
\text { Doctor to be clear, specific and honest in his / her } \\
\text { explanation } \\
\text { Seeing doctor quickly and easily when in need } \\
\text { Being involved in the decision-making process in } \\
\text { choosing any tests or... }\end{array}$} & $197(82.4 \%)$ & $73(30.5 \%)$ & $0.19(0.42)$ \\
\hline & & $105(43.9 \%)$ & $18(7.5 \%)$ & $0.12(0.47)$ \\
\hline & & $132(55.2 \%)$ & $28(11.7 \%)$ & $0.22(0.67)$ \\
\hline & & $149(62.6 \%)$ & $39(16.4 \%)$ & $0.30(0.76)$ \\
\hline & & $143(59.8 \%)$ & $27(11.3 \%)$ & $0.19(0.60)$ \\
\hline & $\begin{array}{c}\text { Cooperation and communication among health care } \\
\text { staff }\end{array}$ & $158(66.1 \%)$ & $34(14.2 \%)$ & $0.26(0.69)$ \\
\hline & $\begin{array}{c}\text { Sincere interest and empathy from the nurses looking } \\
\text { after the person ... }\end{array}$ & $153(64.0 \%)$ & $17(7.1 \%)$ & $0.13(0.51)$ \\
\hline & $\begin{array}{l}\text { Nurses to explain treatment or care that is being given } \\
\text { to the person ... }\end{array}$ & $158(66.1 \%)$ & $20(8.4 \%)$ & $0.13(0.45)$ \\
\hline & $\begin{array}{l}\text { Nurses to promptly attend to the discomfort and pain } \\
\text { of the person ... }\end{array}$ & $151(63.5 \%)$ & $25(10.5 \%)$ & $0.17(0.54)$ \\
\hline \multirow[t]{8}{*}{ Information } & \multirow{2}{*}{$\begin{array}{l}\text { Information about the current status of the illness of the } \\
\text { person I am ... }\end{array}$} & $206(86.2 \%)$ & $109(45.6 \%)$ & $0.33(0.53)$ \\
\hline & & $175(73.8 \%)$ & $43(18.1 \%)$ & $0.37(0.84)$ \\
\hline & \multirow{2}{*}{$\begin{array}{c}\text { Information about tests and treatment } \\
\text { Information about caring for the person with cancer ... } \\
\text { Guidelines or information about complementary and } \\
\text { alternative medicine }\end{array}$} & $\begin{array}{l}176(74.0 \%) \\
167(69.9 \%)\end{array}$ & $\begin{array}{l}45(18.9 \%) \\
41(17.2 \%)\end{array}$ & $\begin{array}{l}0.36(0.80) \\
0.31(0.74)\end{array}$ \\
\hline & & $115(48.5 \%)$ & $33(13.9 \%)$ & $0.27(0.74)$ \\
\hline & $\begin{array}{l}\text { Information about hospitals or clinics and physicians } \\
\text { who treat cancer }\end{array}$ & $156(65.8 \%)$ & $37(15.6 \%)$ & $0.24(0.62)$ \\
\hline & $\begin{array}{c}\text { Information about financial support for medical } \\
\text { expenses ... }\end{array}$ & $165(69.6 \%)$ & $70(29.5 \%)$ & $0.59(1.02)$ \\
\hline & $\begin{array}{c}\text { Help with communication with the person I am caring } \\
\text { for and/or ... }\end{array}$ & $116(49.0 \%)$ & $25(10.6 \%)$ & $0.15(0.48)$ \\
\hline & $\begin{array}{c}\text { Information about caregiving-related stress } \\
\text { management }\end{array}$ & $121(51.1 \%)$ & $46(19.4 \%)$ & $0.31(0.71)$ \\
\hline \multirow[t]{7}{*}{ Hospital facilities } & \multirow{2}{*}{$\begin{array}{l}\text { A designated hospital staff member who would be able } \\
\text { to provide... }\end{array}$} & $178(74.5 \%)$ & $84(35.2 \%)$ & $0.28(0.54)$ \\
\hline & & $128(54.2 \%)$ & $46(19.5 \%)$ & $0.37(0.84)$ \\
\hline & Guidance about hospital facilities and services & $150(63.6 \%)$ & $36(15.3 \%)$ & $0.25(0.67)$ \\
\hline & Need for space reserved for caregivers & $116(49.2 \%)$ & $40(17.0 \%)$ & $0.32(0.79)$ \\
\hline & $\begin{array}{l}\text { A visiting nurse service for the home of the person I am } \\
\text { caring for }\end{array}$ & $61(25.6 \%)$ & $27(11.3 \%)$ & $0.21(0.68)$ \\
\hline & $\begin{array}{c}\text { Opportunity to share experiences or information with } \\
\text { other caregivers }\end{array}$ & $84(35.6 \%)$ & $31(13.1 \%)$ & $0.20(0.57)$ \\
\hline & $\begin{array}{l}\text { Welfare services (e.g., psychological counselling) for } \\
\text { caregivers }\end{array}$ & $98(41.5 \%)$ & $48(20.3 \%)$ & $0.33(0.73)$ \\
\hline \multirow[t]{7}{*}{ Practical Support } & \multirow[b]{2}{*}{$\begin{array}{c}\text { Transportation service for getting to and from the } \\
\text { hospital }\end{array}$} & $207(86.6 \%)$ & $120(50.2 \%)$ & $0.53(0.73)$ \\
\hline & & $146(61.9 \%)$ & $51(21.6 \%)$ & $0.46(0.97)$ \\
\hline & \multirow{2}{*}{$\begin{array}{l}\text { Treatment near home } \\
\text { Lodging near hospital where the person I am caring for } \\
\text { is treated }\end{array}$} & $157(67.1 \%)$ & $86(36.8 \%)$ & $0.85(1.22)$ \\
\hline & & $181(76.7 \%)$ & $57(24.2 \%)$ & $0.57(1.08)$ \\
\hline & \multirow{2}{*}{$\begin{array}{c}\text { Help with economic burden caused by cancer } \\
\text { Someone to help me with housekeeping and/or } \\
\text { childcare }\end{array}$} & $138(58.5 \%)$ & $75(31.8 \%)$ & $0.62(1.03)$ \\
\hline & & $83(34.9 \%)$ & $41(17.2 \%)$ & $0.34(0.83)$ \\
\hline & $\begin{array}{l}\text { Assisted care in hospital or at the home of the person I } \\
\text { am caring for }\end{array}$ & $71(29.8 \%)$ & $31(13.0 \%)$ & $0.24(0.71)$ \\
\hline \multicolumn{2}{|c|}{ All above items (excludes religious/spiritual domain) } & $230(96.2 \%)$ & $171(71.6 \%)$ & $0.33(0.44)$ \\
\hline
\end{tabular}


In terms of single items, the most frequently endorsed needs were lodging near hospital (76.7\%); information about tests and treatment (74.0\%); information about the current status of the illness (73.8\%); and concerns about the person being cared for $(71.2 \%$; see Table 2). The most frequently reported unmet needs were treatment near home (36.8\%), help with economic burden caused by cancer (31.8\%), and concerns about the person being cared for $(31.8 \%)$. Mean degree of unmet need for each item ranged from 0.12 to 0.85 , with treatment near home $(\mathrm{M}=0.85, \mathrm{SD}=1.22)$, help with economic burden $(\mathrm{M}=0.62$, $\mathrm{SD}=1.03)$, information about financial support for medical expenses $(\mathrm{M}=0.59, \mathrm{SD}=1.02)$, lodging near hospital $(\mathrm{M}=0.57, \mathrm{SD}=1.08)$, and concerns about the person being cared for $(\mathrm{M}=0.54, \mathrm{SD}=0.91)$ being the items with the highest mean degree of unmet need.

\subsection{Comparing Overall and Domains of Unmet Need}

Logistic regression analyses suggested that overall unmet need was more frequently reported by female $(\mathrm{OR}=2.12,95 \% \mathrm{CI}=1.18,3.79)$ and younger $(\mathrm{OR}=0.58,95 \% \mathrm{CI}=0.34,0.99)$ caregivers. Practical support was the most commonly reported domain of need for all but a few sub-groups (see Table 3). Unmet health and psychology needs were significantly less likely to be reported by caregivers of breast cancer patients $(\mathrm{OR}=0.43,95 \% \mathrm{CI}=0.20,0.94)$ and older caregivers $(\mathrm{OR}=0.53,95 \% \mathrm{CI}=0.28,1.00)$. Unmet healthcare staff needs were more frequently reported by younger $(\mathrm{OR}=0.35,95 \% \mathrm{CI}=0.17,0.70)$ and female caregivers $(\mathrm{OR}=1.99,95 \% \mathrm{CI}=1.09,3.79)$. Unmet family and social support needs were more frequently reported by younger caregivers $(\mathrm{OR}=0.35,95 \% \mathrm{CI}=0.17,0.71)$.

\subsection{Comparing Single Item Unmet Needs}

Unmet needs related to 'treatment near home' and having unaddressed 'concerns about the person being cared for' were consistently reported by at least $20 \%$ of participants within each demographic and cancer type group (Tables 4 and 5). Overall proportions of unmet needs were relatively stable across demographic and cancer type groups, however, there were some noteworthy exceptions.

Caregivers based in outer regional and remote areas most frequently reported an un-met need for 'treatment near home' (41.8\%), 'help with economic burden' $(33.0 \%)$, 'information about financial support' $(30.4 \%)$, 'concerns about the person I provide care for $(29.7 \%)$, and lodging near hospital $(25.0 \%)$. Caregivers from inner regional areas had a similar pattern, and in addition, at least a quarter of caregivers from inner regional areas reported an unmet need for help with 'my own health problems' (32.3\%), and 'help with my own relaxation and my personal life' $(25.0 \%)$.

For females, 'information about financial support' (32.4\%), 'my own health problems' $(31.0 \%)$, 'lodging near hospital where the person I am caring for is treated' $(27.3 \%)$, and 'feelings of vague anxiety' (26.2\%) were reported as unmet by over a quarter of the sample, whereas less than a quarter of males reported these items.

Among caregivers below the age of 68 , at least a quarter reported an unmet need for 'help with economic burden', 'information about financial support for medical expenses', 'lodging near hospital', 'concerns about the person with cancer', and 'feelings of vague anxiety', whereas $20 \%$ or less of the oldest group ( $>68$ years) reported these items as unmet. For the youngest caregiver group ( $\leq 57$ years), at least a quarter reported 'help with my own relaxation and my personal life' (32.4\%), 'transportation service for getting to and from the hospital' (27.4\%), 'someone to help with housekeeping and/or childcare' (27.4\%), and 'help with difficulties in family relationships' (25.7\%), but these were less frequent among the older age groups (for specific details refer to Table 4). The most common unmet need for caregivers aged over 68 years was help with 'my own health problems' (34.7\%), whereas this was less frequent in younger caregivers ( $26.0 \%$ of caregivers $\leq 57$ years of age; $24.7 \% \geq 58$ and $\leq 68$ years). 
Table 3. Frequency of caregiver unmet need in CNAT-C domains according to patient cancer type and caregiver demographics.

\begin{tabular}{|c|c|c|c|c|c|c|c|c|c|c|c|c|c|c|c|c|c|c|c|}
\hline \multirow[t]{2}{*}{$\begin{array}{l}\text { CNAT-C } \\
\text { Domain }\end{array}$} & \multirow{2}{*}{$\begin{array}{c}\begin{array}{l}\text { Overall } \\
\text { Sample }\end{array} \\
\%(n) \\
\end{array}$} & \multicolumn{2}{|c|}{ Gender } & \multicolumn{3}{|c|}{ Age } & \multicolumn{2}{|c|}{ SES } & \multicolumn{2}{|c|}{ Remoteness } & \multicolumn{3}{|c|}{ Time since Diagnosis } & \multicolumn{6}{|c|}{ Cancer Type } \\
\hline & & $\begin{array}{l}\text { Male } \\
\%(n)\end{array}$ & $\begin{array}{l}\text { Female } \\
\%(n)\end{array}$ & $\begin{array}{l}\leq 57 \\
\text { Years } \\
\%(n)\end{array}$ & $\begin{array}{l}\geq 58 \& \leq 68 \\
\text { Years } \%(n)\end{array}$ & $\begin{array}{l}>68 \\
\text { Years } \\
\%(n)\end{array}$ & $\begin{array}{l}<50 \\
\text { Per- } \\
\text { centile } \\
\%(n)\end{array}$ & $\begin{array}{l}\geq 50 \\
\begin{array}{c}\geq \text { Per- } \\
\text { centile } \\
\%(n)\end{array}\end{array}$ & $\begin{array}{l}\text { Inner } \\
\text { Re- } \\
\text { gional } \\
\%(n)\end{array}$ & $\begin{array}{c}\text { Outer re- } \\
\text { gional/Remote } \\
\%(n)\end{array}$ & $\begin{array}{l}\text { 0-6 } \\
\text { Months } \\
\%(n)\end{array}$ & 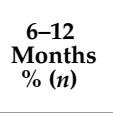 & $\begin{array}{l}>12 \\
\text { Months } \\
\%(n)\end{array}$ & $\begin{array}{l}\text { Breast } \\
\%(n)\end{array}$ & $\underset{(n)}{\operatorname{Skin}} \%$ & $\begin{array}{l}\text { Head } \\
\& \\
\text { Neck } \\
\%(n)\end{array}$ & $\begin{array}{l}\text { Prostate } \\
\%(n)\end{array}$ & $\begin{array}{l}\text { Gynae } \\
\%(n)\end{array}$ & $\begin{array}{l}\text { Other } \\
\%(n)\end{array}$ \\
\hline Practical & $50.2 \%(120)$ & $46.2 \%$ & $52.1 \%$ & $56.8 \%$ & $48.2 \%(41)$ & $\begin{array}{l}44.7 \% \\
(34)\end{array}$ & $\begin{array}{l}50.3 \% \\
(99)\end{array}$ & $51.2 \%$ & $52.4 \%$ & $\begin{array}{l}47.8 \% \\
(54)\end{array}$ & $49.4 \%$ & $\begin{array}{l}48.3 \% \\
(28)\end{array}$ & $51.1 \%$ & $\begin{array}{l}43.2 \% \\
(19)\end{array}$ & $52.9 \%$ & $\begin{array}{l}48.0 \% \\
(12)\end{array}$ & $50.0 \%$ & $52.4 \%$ & $50.7 \%$ \\
\hline $\begin{array}{l}\text { support } \\
\text { Health and } \\
\text { psychological }\end{array}$ & $48.5 \%(116)$ & $\begin{array}{c}41.8 \% \\
(38)\end{array}$ & $\begin{array}{c}52.1 \% \\
(76)\end{array}$ & $\begin{array}{l}56.8 \% \\
(42)\end{array}$ & $49.4 \%(42)$ & $\begin{array}{l}(34) \\
40.8 \% \\
(31)\end{array}$ & $\begin{array}{l}49.8 \% \\
(98)\end{array}$ & $\begin{array}{c}(21) \\
43.9 \% \\
(18)\end{array}$ & $\begin{array}{c}(65) \\
50.8 \% \\
(63)\end{array}$ & $\begin{array}{l}(54) \\
46.9 \% \\
(53)\end{array}$ & $\begin{array}{c}(42) \\
49.4 \% \\
(42)\end{array}$ & $\begin{array}{c}(28) \\
41.4 \% \\
(24)\end{array}$ & $\begin{array}{l}51.1 \% \\
(47)\end{array}$ & $\begin{array}{c}(19) \\
34.1 \% \\
(15)\end{array}$ & $\begin{array}{l}(18) \\
41.2 \% \\
(14)\end{array}$ & $\begin{array}{l}(12) \\
56.0 \% \\
(14)\end{array}$ & $\begin{array}{l}(14) \\
50.0 \% \\
(14)\end{array}$ & $\begin{array}{c}(11) \\
42.9 \% \\
(9)\end{array}$ & $\begin{array}{l}(39) \\
5.8 \% \\
(43)\end{array}$ \\
\hline Information & $45.6 \%(109)$ & $\begin{array}{l}40.7 \% \\
(37)\end{array}$ & $\begin{array}{l}48.0 \% \\
(70)\end{array}$ & $\begin{array}{l}51.4 \% \\
(38)\end{array}$ & $48.2 \%(41)$ & $\begin{array}{l}36.8 \% \\
(28)\end{array}$ & $\begin{array}{l}48.2 \% \\
(95)\end{array}$ & $\begin{array}{c}34.2 \% \\
(14)\end{array}$ & $\begin{array}{l}48.4 \% \\
(60)\end{array}$ & $\begin{array}{l}42.5 \% \\
(48)\end{array}$ & $\begin{array}{c}49.4 \% \\
(42)\end{array}$ & $\begin{array}{c}44.8 \% \\
(26)\end{array}$ & $\begin{array}{l}40.2 \% \\
(37)\end{array}$ & $\begin{array}{l}40.9 \% \\
(18)\end{array}$ & $\begin{array}{l}44.1 \% \\
(15)\end{array}$ & $\begin{array}{l}48.1 \% \\
(12)\end{array}$ & $\begin{array}{l}42.9 \% \\
(12)\end{array}$ & $\begin{array}{l}38.1 \% \\
(8)\end{array}$ & $\begin{array}{c}46.8 \% \\
(36)\end{array}$ \\
\hline $\begin{array}{l}\text { Hospital } \\
\text { facilities and } \\
\text { services }\end{array}$ & $35.2 \%(84)$ & $\begin{array}{l}28.6 \% \\
(26)\end{array}$ & $\begin{array}{l}39.0 \% \\
(57)\end{array}$ & $\begin{array}{l}37.8 \% \\
(28)\end{array}$ & $37.7 \%(32)$ & $\begin{array}{l}29.0 \% \\
(22)\end{array}$ & $\begin{array}{c}36.0 \% \\
(71)\end{array}$ & $\begin{array}{c}31.7 \% \\
(13)\end{array}$ & $\begin{array}{l}36.3 \% \\
(45)\end{array}$ & $\begin{array}{l}33.6 \% \\
(38)\end{array}$ & $\begin{array}{c}35.3 \% \\
(30)\end{array}$ & $\begin{array}{c}31.0 \% \\
(18)\end{array}$ & $\begin{array}{c}34.8 \% \\
(32)\end{array}$ & $\begin{array}{l}27.3 \% \\
(12)\end{array}$ & $\begin{array}{c}35.3 \% \\
(12)\end{array}$ & $\begin{array}{c}36.0 \% \\
(9)\end{array}$ & $\begin{array}{l}28.6 \% \\
(8)\end{array}$ & $\begin{array}{l}42.9 \% \\
(9)\end{array}$ & $\begin{array}{c}35.1 \% \\
(27)\end{array}$ \\
\hline Family/social & $32.6 \%(78)$ & $27.5 \%$ & $34.9 \%$ & $47.3 \%$ & $31.8 \%(27)$ & $19.7 \%$ & $35.0 \%$ & $22.0 \%$ & $35.5 \%$ & $30.1 \%$ & $27.1 \%$ & $34.5 \%$ & $34.8 \%$ & $27.3 \%$ & $20.6 \%$ & $28.0 \%$ & $25.0 \%$ & $23.8 \%$ & $42.9 \%$ \\
\hline $\begin{array}{l}\text { support } \\
\text { Health-care } \\
\text { staff }\end{array}$ & $30.5 \%(73)$ & $\begin{array}{l}(25) \\
2.0 \% \\
(20)\end{array}$ & $\begin{array}{c}(51) \\
36.3 \% \\
(53)\end{array}$ & $\begin{array}{l}(35) \\
39.2 \% \\
(29)\end{array}$ & $32.9 \%(28)$ & $\begin{array}{l}(15) \\
19.7 \% \\
(15)\end{array}$ & $\begin{array}{c}(69) \\
31.5 \% \\
(62)\end{array}$ & $\begin{array}{c}(9) \\
26.8 \% \\
(11)\end{array}$ & $\begin{array}{c}(44) \\
30.7 \% \\
(38)\end{array}$ & $\begin{array}{l}(34) \\
31.0 \% \\
(35)\end{array}$ & $\begin{array}{c}(23) \\
23.5 \% \\
(20)\end{array}$ & $\begin{array}{c}(20) \\
32.8 \% \\
(19)\end{array}$ & $\begin{array}{l}(32) \\
33.7 \% \\
(31)\end{array}$ & $\begin{array}{c}(12) \\
22.7 \% \\
(10)\end{array}$ & $\begin{array}{l}(7) \\
41.2 \% \\
(14)\end{array}$ & $\begin{array}{c}(7) \\
24.6 \% \\
(6)\end{array}$ & $\begin{array}{c}(7) \\
25.0 \% \\
(7)\end{array}$ & $\begin{array}{l}(5) \\
14.3 \% \\
(3)\end{array}$ & $\begin{array}{l}(33) \\
36.4 \% \\
(28)\end{array}$ \\
\hline $\begin{array}{c}\text { All } \\
\text { domains-any } \\
\text { unmet need }\end{array}$ & $71.6 \%(171)$ & $\begin{array}{l}61.5 \% \\
(56)\end{array}$ & $\begin{array}{l}77.4 \% \\
(113)\end{array}$ & $\begin{array}{l}78.4 \% \\
(58)\end{array}$ & $70.6 \%(60)$ & $\begin{array}{l}65.8 \% \\
(50)\end{array}$ & $\begin{array}{l}73.1 \% \\
(144)\end{array}$ & $\begin{array}{c}65.9 \% \\
(27)\end{array}$ & $\begin{array}{l}71.8 \% \\
(89)\end{array}$ & $\begin{array}{l}71.7 \% \\
(81)\end{array}$ & $\begin{array}{c}72.9 \% \\
(62)\end{array}$ & $\begin{array}{c}72.4 \% \\
(42)\end{array}$ & $\begin{array}{l}68.5 \% \\
(63)\end{array}$ & $\begin{array}{l}63.6 \% \\
(28)\end{array}$ & $\begin{array}{l}76.5 \% \\
(26)\end{array}$ & $\begin{array}{l}72.0 \% \\
(18)\end{array}$ & $\begin{array}{l}71.4 \% \\
(20)\end{array}$ & $\begin{array}{l}61.9 \% \\
(13)\end{array}$ & $\begin{array}{c}74.0 \% \\
(57)\end{array}$ \\
\hline
\end{tabular}

Abbreviation: Gynae = Gynaecological 
Table 4. Frequency of unmet need ${ }^{\mathbf{1}}$ by age, gender, and area level characteristics.

\begin{tabular}{|c|c|c|c|c|c|c|c|c|c|c|}
\hline \multirow[b]{2}{*}{ CNAT-C Item (25 Most Frequent Overall) } & \multirow{2}{*}{$\begin{array}{c}\begin{array}{c}\text { Overall } \\
\text { Sample }\end{array} \\
\% \text { (Rank) }\end{array}$} & \multicolumn{2}{|c|}{ Gender } & \multicolumn{3}{|c|}{ Age } & \multicolumn{2}{|c|}{ Remoteness } & \multicolumn{2}{|c|}{$\begin{array}{l}\text { Area-Level Disadvantage } \\
\text { (SEIFA) }\end{array}$} \\
\hline & & $\begin{array}{c}\text { Male } \\
\% \text { (Rank) }\end{array}$ & $\begin{array}{c}\text { Female } \\
\% \text { (Rank) }\end{array}$ & $\begin{array}{c}\leq 57 \text { Years \% } \\
\text { (Rank) }\end{array}$ & $\begin{array}{l}\geq 58 \& \leq 68 \\
\text { Years\% } \\
\text { (Rank) }\end{array}$ & $\begin{array}{c}>68 \text { Years \% } \\
\text { (Rank) }\end{array}$ & $\begin{array}{c}\text { Inner } \\
\text { Regional } \\
(n=124) \\
\%(\text { Rank })\end{array}$ & $\begin{array}{c}\text { Outer } \\
\text { Regional \& } \\
\text { Remote } \\
(n=113) \\
\% \text { (Rank) }\end{array}$ & $\begin{array}{l}<50 \text { Percentile } \\
(n=197) \\
\% \text { (Rank) }\end{array}$ & $\begin{array}{l}\geq 50 \text { Percentile } \\
\quad(n=41) \\
\quad \% \text { (Rank) }\end{array}$ \\
\hline & $\begin{array}{l}36.8 \%(1) \\
31.8 \%(2)\end{array}$ & $\begin{array}{l}29.2 \%(3) \\
34.4 \%(1)\end{array}$ & $\begin{array}{l}41.3 \%(1) \\
29.9 \%(4)\end{array}$ & $\begin{array}{l}41.7 \%(3) \\
43.8 \%(1)\end{array}$ & $\begin{array}{l}39.3 \%(1) \\
30.6 \%(4)\end{array}$ & $\begin{array}{l}27.0 \%(2) \\
20.3 \%(3)\end{array}$ & $\begin{array}{l}32.0 \%(3) \\
30.3 \%(4)\end{array}$ & $\begin{array}{l}41.8 \%(1) \\
33.0 \%(2)\end{array}$ & $\begin{array}{l}36.3 \%(1) \\
32.0 \%(3)\end{array}$ & $\begin{array}{l}40.0 \%(1) \\
31.7 \%(2)\end{array}$ \\
\hline \multirow{2}{*}{$\begin{array}{l}\text { Help with economic burden caused by cancer } \\
\text { Concerns about the person I provide care for } \\
\text { Information about financial support for medical } \\
\text { expenses, from ... }\end{array}$} & $31.8 \%$ (2) & $32.6 \%(2)$ & $31.0 \%$ (3) & $42.5 \%$ (2) & $32.9 \%(3)$ & $20.0 \%(4)$ & $33.9 \%(1)$ & $29.7 \%(4)$ & $32.5 \%$ (2) & $29.3 \%$ (3) \\
\hline & $29.5 \%(3)$ & $24.4 \%(4)$ & $32.4 \%(2)$ & $36.1 \%(4)$ & $34.1 \%(2)$ & $17.1 \%(8)$ & $28.5 \%(5)$ & $30.4 \%(3)$ & $29.7 \%(5)$ & $29.3 \%(3)$ \\
\hline \multirow{2}{*}{$\begin{array}{l}\text { My own health problems } \\
\text { Lodging near hospital where the person I am caring } \\
\text { for is treated }\end{array}$} & $28.0 \%(4)$ & $22.5 \%(6)$ & $31.0 \%(3)$ & $26.0 \%(8)$ & $24.7 \%(7)$ & $34.7 \%(1)$ & $32.3 \%(2)$ & $23.4 \%(6)$ & $29.9 \%(4)$ & $19.5 \%(5)$ \\
\hline & $24.2 \%(5)$ & $17.6 \%(12)$ & $27.3 \%(5)$ & $32.9 \%(5)$ & $25.0 \%(6)$ & $14.7 \%(11)$ & $23.8 \%(8)$ & $25.0 \%$ (5) & $23.2 \%(8)$ & $29.3 \%$ (3) \\
\hline \multirow{2}{*}{$\begin{array}{l}\text { Feelings of vague anxiety } \\
\text { Help with my own relaxation and my personal life } \\
\text { Transportation service for getting to and from the } \\
\text { hospital }\end{array}$} & $\begin{array}{l}23.7 \%(6) \\
22.6 \%(7)\end{array}$ & $\begin{array}{l}19.1 \%(8) \\
18.7 \%(9)\end{array}$ & $\begin{array}{l}26.2 \%(6) \\
24.7 \%(8)\end{array}$ & $\begin{array}{l}26.0 \%(8) \\
32.4 \%(6)\end{array}$ & $\begin{array}{l}25.9 \%(5) \\
23.5 \%(9)\end{array}$ & $\begin{array}{l}18.7 \%(6) \\
11.8 \%(16)\end{array}$ & $\begin{array}{l}24.2 \%(7) \\
25.0 \%(6)\end{array}$ & $\begin{array}{l}23.4 \%(6) \\
20.4 \%(9)\end{array}$ & $\begin{array}{l}25.8 \%(6) \\
23.9 \%(7)\end{array}$ & $\begin{array}{l}14.6 \%(8) \\
17.1 \%(7)\end{array}$ \\
\hline & $21.6 \%(8)$ & $24.2 \%(5)$ & $20.3 \%(14)$ & $27.4 \%(7)$ & $21.2 \%(11)$ & $14.9 \%(10)$ & $22.0 \%(9)$ & $20.7 \%(8)$ & $21.5 \%(10)$ & $22.5 \%(4)$ \\
\hline \multirow{5}{*}{$\begin{array}{c}\text { Welfare services (e.g., psychological counselling) for } \\
\text { caregivers } \\
\text { Feelings of anger, irritability or nervousness } \\
\text { A designated hospital staff member who would be } \\
\text { able to provide... } \\
\text { Information about caregiving-related stress } \\
\text { management } \\
\text { Information about tests and treatment }\end{array}$} & $20.3 \%(9)$ & $16.7 \%(14)$ & $22.2 \%(10)$ & $23.3 \%(13)$ & $20.2 \%(12)$ & $17.3 \%(7)$ & $19.5 \%(12)$ & $21.6 \%(7)$ & $21.1 \%(12)$ & $17.1 \%(7)$ \\
\hline & $20.0 \%(10)$ & $16.9 \%(13)$ & $22.2 \%(10)$ & $24.7 \%(10)$ & $21.2 \%(11)$ & $13.5 \%(12)$ & $20.3 \%(11)$ & $19.8 \%(10)$ & $21.8 \%(9)$ & $12.2 \%(9)$ \\
\hline & $19.5 \%(11)$ & $13.3 \%(17)$ & $23.6 \%(9)$ & $21.9 \%(14)$ & $23.8 \%(8)$ & $12.0 \%(15)$ & $22.0 \%(9)$ & $17.1 \%(15)$ & $19.9 \%(14)$ & $18.0 \%(6)$ \\
\hline & $19.4 \%(12)$ & $17.8 \%$ (11) & $20.0 \%(15)$ & $23.6 \%(12)$ & $20.0 \%(13)$ & $15.8 \%(9)$ & $22.0 \%(9)$ & $17.0 \%(16)$ & $21.0 \%(13)$ & $12.2 \%(9)$ \\
\hline & $18.9 \%(13)$ & $8.8 \%(26)$ & $24.8 \%(7)$ & $14.9 \%(24)$ & $22.4 \%(10)$ & $18.7 \%(6)$ & $18.7 \%(13)$ & $19.5 \%(12)$ & $21.4 \%(11)$ & $7.3 \%(12)$ \\
\hline \multirow{2}{*}{$\begin{array}{l}\text { Depression } \\
\text { Loneliness or feelings of isolation } \\
\text { Information about the current status of the illness of } \\
\text { the person I am ... }\end{array}$} & $\begin{array}{l}18.3 \%(14) \\
18.2 \%(15)\end{array}$ & $\begin{array}{l}18.0 \%(10) \\
20.2 \%(7)\end{array}$ & $\begin{array}{l}18.1 \%(17) \\
17.2 \%(18)\end{array}$ & $\begin{array}{l}21.9 \%(14) \\
24.7 \%(10)\end{array}$ & $\begin{array}{l}19.1 \%(14) \\
18.8 \%(15)\end{array}$ & $\begin{array}{l}14.7 \%(11) \\
12.0 \%(15)\end{array}$ & $\begin{array}{l}19.5 \%(12) \\
18.6 \%(14)\end{array}$ & $\begin{array}{l}17.1 \%(15) \\
18.0 \%(13)\end{array}$ & $\begin{array}{l}18.1 \%(17) \\
19.1 \%(15)\end{array}$ & $\begin{array}{l}19.5 \%(5) \\
14.6 \%(8)\end{array}$ \\
\hline & $18.1 \%(16)$ & $11.1 \%(20)$ & $22.1 \%(11)$ & $15.1 \%$ & $21.2 \%(11)$ & $17.3 \%(7)$ & $20.3 \%(11)$ & $16.1 \%(18)$ & $19.9 \%(14)$ & $10.0 \%(10)$ \\
\hline \multirow{2}{*}{$\begin{array}{l}\text { Someone to help me with housekeeping and/or child } \\
\text { care } \\
\text { Information about caring for the person with cancer } \\
\text { (symptom .... }\end{array}$} & $17.2 \%(17)$ & $17.6 \%(12)$ & $16.6 \%(19)$ & $27.4 \%(7)$ & $5.9 \%(26)$ & $19.7 \%(5)$ & $21.0 \%(10)$ & $12.5 \%(25)$ & $16.8 \%(19)$ & $19.5 \%(5)$ \\
\hline & $17.2 \%(18)$ & $9.9 \%(24)$ & $21.2 \%(12)$ & $20.3 \%(17)$ & $18.8 \%(15)$ & $13.2 \%(14)$ & $17.7 \%(15)$ & $16.8 \%(17)$ & $18.8 \%(16)$ & $9.8 \%(11)$ \\
\hline \multirow{2}{*}{$\begin{array}{l}\text { Need for space reserved for caregivers } \\
\text { Seeing doctor quickly and easily when in need } \\
\text { Information about hospitals or clinics and physicians } \\
\text { who treat cancer }\end{array}$} & $\begin{array}{l}17.0 \%(19) \\
16.4 \%(20)\end{array}$ & $\begin{array}{l}14.3 \%(16) \\
9.9 \%(24)\end{array}$ & $\begin{array}{l}18.9 \%(16) \\
20.7 \%(13)\end{array}$ & $\begin{array}{l}12.5 \%(28) \\
18.9 \%(20)\end{array}$ & $\begin{array}{l}22.4 \%(10) \\
19.1 \%(14)\end{array}$ & $\begin{array}{l}13.3 \%(13) \\
11.8 \%(16)\end{array}$ & $\begin{array}{l}16.3 \%(16) \\
13.7 \%(21)\end{array}$ & $\begin{array}{l}17.1 \%(15) \\
19.6 \%(11)\end{array}$ & $\begin{array}{l}16.5 \%(20) \\
17.9 \%(18)\end{array}$ & $\begin{array}{l}19.5 \%(5) \\
9.8 \%(11)\end{array}$ \\
\hline & $15.6 \%(21)$ & $12.2 \%(19)$ & $17.2 \%(18)$ & $20.8 \%(15)$ & $15.3 \%(17)$ & $10.5 \%(18)$ & $13.8 \%(20)$ & $17.9 \%(14)$ & $15.4 \%(22)$ & $17.1 \%(7)$ \\
\hline $\begin{array}{c}\text { Help with difficulties in family relationships after } \\
\text { cancer diagnosis }\end{array}$ & $15.5 \%(22)$ & $14.3 \%(16)$ & $15.1 \%(24)$ & $25.7 \%(9)$ & $14.1 \%(18)$ & $7.9 \%(22)$ & $16.1 \%(17)$ & $15.0 \%(20)$ & $14.7 \%(25)$ & $19.5 \%(5)$ \\
\hline \multirow{2}{*}{$\begin{array}{l}\text { Guidance about hospital facilities and services } \\
\text { Help with difficulties in interpersonal relationships } \\
\text { after cancer ... }\end{array}$} & $15.3 \%(23)$ & $11.1 \%(20)$ & $18.1 \%(17)$ & $19.4 \%(18)$ & $18.8 \%(15)$ & $8.0 \%(21)$ & $16.3 \%(16)$ & $14.4 \%(21)$ & $15.0 \%(24)$ & $17.1 \%(7)$ \\
\hline & $15.1 \%(24)$ & $16.5 \%(15)$ & $13.7 \%(26)$ & $24.3 \%(11)$ & $12.9 \%(19)$ & $9.2 \%(20)$ & $14.5 \%(19)$ & $15.9 \%(19)$ & $15.2 \%(23)$ & $14.6 \%(8)$ \\
\hline $\begin{array}{l}\text { Cooperation and communication among health care } \\
\text { staff }\end{array}$ & $14.2 \%(25)$ & $11.0 \%(21)$ & $16.4 \%(20)$ & $16.2 \%(22)$ & $17.7 \%(16)$ & $7.9 \%(22)$ & $12.1 \%(24)$ & $16.8 \%(17)$ & $14.7 \%(25)$ & $12.2 \%(9)$ \\
\hline
\end{tabular}

${ }^{1}$ Unmet need = items with a low, moderate or high need; $\ldots$ = item truncated; Shading represents items where $25 \%$ or more of each group reported this need. 
Table 5. Frequency of unmet needs ${ }^{\mathbf{1}}$ according to patient disease characteristics.

\begin{tabular}{|c|c|c|c|c|c|c|c|c|c|c|}
\hline \multirow[b]{2}{*}{ CNAT-C Item (25 Most Frequent Overall) } & \multirow{2}{*}{$\begin{array}{l}\text { Overall } \\
\text { Sample } \\
\% \text { (Rank) }\end{array}$} & \multicolumn{3}{|c|}{ Time since Patient Diagnosis } & \multicolumn{6}{|c|}{ Cancer Type of Patient } \\
\hline & & $\begin{array}{l}0-6 \text { Months ( } n \\
=85) \\
\% \text { (Rank) }\end{array}$ & $\begin{array}{c}\text { 6-12 Months } \\
(n=58) \\
\%(\text { Rank })\end{array}$ & $\begin{array}{c}>12 \text { Months }(n \\
=92) \\
\% \text { (Rank) }\end{array}$ & $\begin{array}{c}\text { Breast } \\
(n=44) \% \\
(\text { Rank })\end{array}$ & $\begin{array}{c}\text { Skin } \\
(n=34) \\
(\text { Rank })\end{array}$ & $\begin{array}{l}\text { Head \& Neck } \\
\quad(n=25) \\
\%(\text { Rank }) \\
\end{array}$ & $\begin{array}{c}\text { Prostate } \\
(n=28) \\
\% \text { (Rank) }\end{array}$ & $\begin{array}{c}\text { Gynae } \\
(n=21) \% \\
(\text { Rank })\end{array}$ & $\begin{array}{c}\text { Other } \\
(n=77) \% \\
(\text { Rank })\end{array}$ \\
\hline \multirow{2}{*}{$\begin{array}{c}\text { Treatment near home } \\
\text { Help with economic burden caused by cancer } \\
\text { Concerns about the person I provide care for } \\
\text { Information about financial support for medical } \\
\text { expenses ... }\end{array}$} & $36.8 \%(1)$ & $33.3 \%(1)$ & $32.8 \%(2)$ & $40.7 \%(1)$ & $28.6 \%(3)$ & $45.5 \%(1)$ & $40.0 \%(1)$ & $46.2 \%(1)$ & $28.6 \%(2)$ & $33.8 \%(3)$ \\
\hline & $29.5 \%(3)$ & $26.2 \%(4)$ & $29.8 \%(4)$ & $30.4 \%(4)$ & $29.6 \%(2)$ & $30.3 \%(3)$ & $36.0 \%(2)$ & $21.4 \%(5)$ & $19.1 \%(5)$ & $30.3 \%(5)$ \\
\hline \multirow{2}{*}{$\begin{array}{l}\text { My own health problems } \\
\text { Lodging near hospital where the person I am caring } \\
\text { for is treated }\end{array}$} & $28.0 \%(4)$ & $25.0 \%(5)$ & $21.1 \%(9)$ & $33.0 \%(2)$ & $18.6 \%(8)$ & $17.7 \%(12)$ & $28.0 \%(4)$ & $35.7 \%(2)$ & $35.0 \%(1)$ & $31.6 \%(4)$ \\
\hline & $24.2 \%(5)$ & $21.7 \%(7)$ & $24.1 \%(7)$ & $26.4 \%(6)$ & $20.5 \%(7)$ & $29.0 \%(5)$ & $28.0 \%(4)$ & $28.6 \%(3)$ & $19.1 \%(5)$ & $20.8 \%(12)$ \\
\hline \multirow{2}{*}{$\begin{array}{l}\text { Feelings of vague anxiety } \\
\text { Help with my own relaxation and my personal life } \\
\text { Transportation service for getting to and from the } \\
\text { hospital }\end{array}$} & $\begin{array}{l}23.7 \%(6) \\
22.6 \%(7)\end{array}$ & $\begin{array}{l}23.8 \%(6) \\
15.3 \%(15)\end{array}$ & $\begin{array}{l}22.8 \%(8) \\
25.9 \%(5)\end{array}$ & $\begin{array}{l}24.2 \%(7) \\
27.2 \%(5)\end{array}$ & $\begin{array}{c}18.6 \%(8) \\
15.9 \%(11)\end{array}$ & $\begin{array}{l}17.7 \%(12) \\
17.7 \%(12)\end{array}$ & $\begin{array}{l}24.0 \%(5) \\
16.0 \%(8)\end{array}$ & $\begin{array}{l}21.4 \%(5) \\
21.4 \%(5)\end{array}$ & $\begin{array}{l}15.0 \%(6) \\
14.3 \%(7)\end{array}$ & $\begin{array}{l}31.6 \%(4) \\
29.9 \%(6)\end{array}$ \\
\hline & $21.6 \%(8)$ & $19.5 \%(8)$ & $17.2 \%(14)$ & $27.2 \%(5)$ & $20.5 \%(7)$ & $28.1 \%(6)$ & $24.0 \%(5)$ & $14.8 \%(8)$ & $28.6 \%(2)$ & $19.5 \%(13)$ \\
\hline \multirow{3}{*}{$\begin{array}{l}\text { Feelings of anger, irritability or nervousness } \\
\text { A designated hospital staff member who would be } \\
\text { able to provide .... }\end{array}$} & $20.3 \%(9)$ & $16.9 \%(13)$ & $17.5 \%(13)$ & $23.9 \%(8)$ & $16.3 \%(10)$ & $28.1 \%(6)$ & $24.0 \%(5)$ & $10.7 \%(11)$ & $23.8 \%(4)$ & $18.2 \%(15)$ \\
\hline & $20.0 \%(10)$ & $19.3 \%(9)$ & $19.3 \%(11)$ & $20.9 \%(12)$ & $16.3 \%(10)$ & $11.8 \%(16)$ & $29.2 \%(3)$ & $14.3 \%(9)$ & $10.0 \%(8)$ & $25.0 \%(7)$ \\
\hline & $19.5 \%(11)$ & $18.1 \%(11)$ & $17.2 \%(14)$ & $19.8 \%(13)$ & $13.6 \%(13)$ & $21.2 \%(9)$ & $16.7 \%(7)$ & $18.5 \%(6)$ & $14.3 \%(7)$ & $22.1 \%(11)$ \\
\hline $\begin{array}{l}\text { Information about caregiving-related stress } \\
\text { management }\end{array}$ & $19.4 \%(12)$ & $17.9 \%(12)$ & $19.3 \%(11)$ & $19.6 \%(14)$ & $20.5 \%(7)$ & $12.1 \%(15)$ & $16.0 \%(8)$ & $10.7 \%(11)$ & $14.3 \%(7)$ & $25.0 \%(7)$ \\
\hline Information about tests and treatment & $18.9 \%(13)$ & $19.1 \%(10)$ & $17.2 \%(14)$ & $16.3 \%(17)$ & $2.3 \%(18)$ & $26.5 \%(7)$ & $12.0 \%(9)$ & $21.4 \%(5)$ & $9.5 \%(9)$ & $23.7 \%(8)$ \\
\hline \multirow{2}{*}{$\begin{array}{l}\text { Depression } \\
\text { Loneliness or feelings of isolation } \\
\text { Information about the current status of the illness of } \\
\text { the person I am caring for ... } \\
\text { Someone to help me with housekeeping and/or child }\end{array}$} & $\begin{array}{l}18.3 \%(14) \\
18.2 \%(15)\end{array}$ & $\begin{array}{l}13.3 \%(18) \\
10.7 \%(21)\end{array}$ & $\begin{array}{l}19.3 \%(11) \\
21.1 \%(9)\end{array}$ & $\begin{array}{l}22.0 \%(11) \\
23.1 \%(9)\end{array}$ & $\begin{array}{l}18.6 \%(8) \\
20.9 \%(6)\end{array}$ & $\begin{array}{l}17.7 \%(12) \\
11.8 \%(16)\end{array}$ & $\begin{array}{l}28.0 \%(4) \\
28.0 \%(4)\end{array}$ & $\begin{array}{l}11.1 \%(10) \\
3.6 \%(13)\end{array}$ & $\begin{array}{l}15.0 \%(6) \\
15.0 \%(6)\end{array}$ & $\begin{array}{l}18.4 \%(14) \\
22.4 \%(10)\end{array}$ \\
\hline & $18.1 \%(16)$ & $16.7 \%(14)$ & $14.0 \%(18)$ & $18.5 \%(15)$ & $4.6 \%(17)$ & $20.6 \%(10)$ & $16.0 \%(8)$ & $18.5 \%(6)$ & $9.5 \%(9)$ & $22.4 \%(10)$ \\
\hline \multirow{3}{*}{$\begin{array}{l}\text { care } \\
\text { Information about caring for the person with cancer } \\
\text { (symptom management,... } \\
\text { Need for space reserved for caregivers } \\
\text { Seeing doctor quickly and easily when in need } \\
\text { Information about hospitals or clinics and physicians }\end{array}$} & $17.2 \%(18)$ & $15.3 \%(15)$ & $20.7 \%(10)$ & $15.2 \%(19)$ & $4.6 \%(17)$ & $20.6 \%(10)$ & $16.0 \%(8)$ & $21.4 \%(5)$ & $9.5 \%(9)$ & $20.8 \%(12)$ \\
\hline & $\begin{array}{l}17.0 \%(19) \\
16.4 \%(20)\end{array}$ & $\begin{array}{l}16.9 \%(13) \\
10.6 \%(22)\end{array}$ & $\begin{array}{l}10.3 \%(21) \\
24.6 \%(6)\end{array}$ & $\begin{array}{l}19.8 \%(13) \\
16.3 \%(17)\end{array}$ & $\begin{array}{l}11.4 \%(14) \\
9.1 \%(15)\end{array}$ & $\begin{array}{l}12.1 \%(15) \\
20.6 \%(10)\end{array}$ & $\begin{array}{l}20.0 \%(6) \\
8.0 \%(11)\end{array}$ & $\begin{array}{c}0.0 \%(14) \\
10.7 \%(11)\end{array}$ & $\begin{array}{l}23.8 \%(4) \\
10.0 \%(8)\end{array}$ & $\begin{array}{l}23.7 \%(8) \\
23.4 \%(9)\end{array}$ \\
\hline & $15.6 \%(21)$ & $11.9 \%(20)$ & $14.0 \%(18)$ & $17.4 \%(16)$ & $15.9 \%(11)$ & $21.2 \%(9)$ & $8.0 \%(11)$ & $14.3 \%(9)$ & $19.1 \%(5)$ & $13.2 \%(20)$ \\
\hline $\begin{array}{l}\text { Help with difficulties in family relationships after } \\
\text { cancer diagnosis }\end{array}$ & $15.5 \%(22)$ & $10.6 \%(22)$ & $20.7 \%(10)$ & $15.2 \%(19)$ & $15.9 \%(11)$ & $5.9 \%(20)$ & $20.0 \%(6)$ & $17.9 \%(7)$ & $4.8 \%$ (11) & $18.2 \%(15)$ \\
\hline \multirow{3}{*}{$\begin{array}{l}\text { Guidance about hospital facilities and } \\
\text { services } \\
\text { Help with difficulties in interpersonal relationships } \\
\text { after cancer diagnosis } \\
\text { Cooperation and communication among health care } \\
\text { staff }\end{array}$} & $15.3 \%(23)$ & $14.5 \%(16)$ & $14.0 \%(18)$ & $15.2 \%(19)$ & $9.1 \%(15)$ & $18.8 \%(11)$ & $16.0 \%(8)$ & $14.3 \%(9)$ & $9.5 \%(9)$ & $18.4 \%$ (14) \\
\hline & $15.1 \%(24)$ & $12.9 \%(19)$ & $17.2 \%(14)$ & $15.2 \%(19)$ & $18.2 \%(9)$ & $5.9 \%(20)$ & $24.0 \%(5)$ & $7.1 \%(12)$ & $0.0 \%(12)$ & $19.5 \%(13)$ \\
\hline & $14.2 \%(25)$ & $8.2 \%(25)$ & $19.0 \%(12)$ & $14.1 \%(21)$ & $18.2 \%(9)$ & $14.7 \%(14)$ & $8.0 \%(11)$ & $10.7 \%(11)$ & $4.8 \%(11)$ & $14.3 \%(19)$ \\
\hline
\end{tabular}

${ }^{1}$ Unmet need $=$ items with a low, moderate or high need. Abbreviation: Gynae $=$ Gynaecological $; \ldots=$ item truncated.Shading represents items where $25 \%$ or more of each group reported this need. 
In terms of the time since diagnosis, 'help with my own relaxation and my personal life' was reported by over a quarter of caregivers of patients who were diagnosed over six months ago, but was less common within the first six months since diagnosis. In addition, among caregivers of patients diagnosed over twelve months ago, 'transportation service for getting to and from the hospital' was reported by $27.2 \%$. Help with 'my own health problems' was reported by less than $20 \%$ of caregivers of patients with breast cancer and skin cancer, even though it was reported by at least a quarter of caregivers of patients with other cancer types (frequencies ranged between $28.0 \%$ and $35.7 \%$, for details refer to Table 5). 'Help with economic burden caused by cancer' was reported by $10.7 \%$ of caregivers of patients with prostate cancer, whereas it was reported by more than a quarter of caregivers of patients with the other cancer types. Similarly, 'information about financial support' was reported as unmet by more than a quarter of most caregivers, except for those caring for patients with gynaecological or prostate cancer. In general, 'feelings of vague anxiety', 'help with my own relaxation and personal life', and 'information about caregiving-related stress management' were reported as unmet by less than a quarter of caregivers. However, among those caring for a patient with 'other' cancer types (consisting of the least common cancers in this sample), these items were endorsed by at least $25 \%$. These frequencies still held even after removing colorectal cancer, non-Hodgkins lymphoma, and lung cancer from the 'other' cancer group, since they are relatively common cancers although not wellrepresented in this sample. The same three items representing psychological or emotional distress were endorsed by at least $28 \%$ of caregivers of the remaining 'other' cancer types (such as brain, oesophageal, stomach, bladder cancer). 'Feelings of anger, irritability or nervousness', 'loneliness or feelings of isolation', and 'depression' were reported by more than a quarter of caregivers of patients with head and neck cancer, yet was less frequent when considering the sample overall.

\section{Discussion}

The current findings provide valuable insight into the areas where caregivers caring for cancer patients in regional and remote Australia require support. Needs that were associated with treatment, staff and facilities were generally well-addressed in this regional and remote sample, whereas a large degree of unmet need was evident in areas relating to practical and psychological support. These findings are consistent with previous studies on patient $[27,28]$ and caregiver $[29,30]$ cohorts that suggest higher levels of satisfaction with health care and facilities during treatment, but poorer access to peripheral support. These findings support calls for better provision of psychosocial and practical support following treatment when regional and remote patients and caregivers return to isolated settings [31]. Unsurprisingly, accommodation near treatment was an important, but well-addressed, need in this cohort of participants recruited through subsided lodges available for the purpose of providing accommodation close to treatment.

Gender differences in unmet need were evident, with female caregivers reporting higher levels of unmet need than males particularly when it came to interactions with healthcare staff. Females were almost two times more likely to report one or more needs in this domain. Previous research has frequently found female caregivers to report greater unmet needs than males $[9,11,12,32,33]$. This may be interconnected with the greater emotional distress and adverse outcomes reported by female caregivers compared to males, which is another consistent finding in the literature [32,34-36]. Although the mechanisms underlying the gender difference are not well understood, theoretical explanations include the impact of internalized traditional gender roles on psychological processes and behaviour [34]. For example, men may be less likely to perceive or disclose their needs for help, compared to women [37]. In addition, men may tend to perceive the caring role as a competency task leading to a positive appraisal and self-mastery and esteem response [32,38], whereas women may tend to hold high internal expectations and a sense of moral obligation related to their caring role, leading to over-responsibility, dys- 
functional self-sacrifice, and an absence of self-care $[32,33,38,39]$. These theories require further evaluation.

Overall, our findings suggested that younger caregivers are slightly more likely to experience unmet needs; however, in terms of specific needs, older caregivers more often experienced unmet needs relating to maintaining their own health. Conversely, younger caregivers were slightly to moderately more likely to report a need for psychosocial support. This may reflect the tendency for people to report slightly better mental health related quality of life and slightly poorer physical quality of life as they age [40]. Furthermore, younger caregivers may be more likely to be employed and have other social roles and family responsibilities, which could lead to them experiencing a heavier load from additional caregiving responsibilities. They may have comparatively less experience with serious illnesses and navigating the health system, and be less likely to consider caregiving a normative task for their age-group. These factors may thus contribute to younger caregivers experiencing greater psychological distress than older caregivers [41].

Descriptive differences in frequencies of unmet need for single items according to cancer type suggest a trend that caregivers of patients with head and neck cancer and other less common cancers who live in regional or remote areas, may benefit from additional psychosocial support. Previous research indicates the psychological and emotional needs of caregivers of head and neck patients are consistently high over time, possibly related to a lack of support, and the complex patient care needs and functional difficulties posttreatment [42]. Similarly, there is evidence that patients with rare cancers may have poorer psychosocial outcomes than the general cancer population [43], which could impact caregivers, however, findings around this are mixed [44]. There is limited empirical research into the psychosocial well-being and needs of caregivers of patients with rare cancers in general [45], highlighting a need for future research on the topic.

\subsection{Recommendations}

The current findings suggest that regional and remote cancer caregivers need extra support to cope, not only with practical issues such as travel, financial losses and household upkeep, but also their mental well-being and concerns regarding the patient they care for. Although providing treatment and counselling facilities closer to home may not be a viable strategy, tele-health and community-based services hold promise in partly addressing these needs $[31,46]$.

Some caregiver and patient characteristics may be important to consider in planning such interventions. That is, tailoring supportive care to target individual and situational characteristics is likely to be as important for caregivers as it is when addressing patient support needs $[47,48]$. According to the current findings, this may include providing younger caregivers with more information about financial support, while assisting older caregivers with managing their own health. The unique experiences of younger female caregivers should be considered in caregiver-health professional interactions to ensure needs are met in terms of respect and quality communication regarding the patient. Finally, providing caregivers with an opportunity to rest and recover is essential for maintaining their capacity to care for their loved ones [49]. According to the current findings, the respite and emotional needs of caregivers appear to be particularly important in the period 6 to 12 months after diagnosis.

\subsection{Strengths and Limitations}

This study utilised a relatively large sample of caregivers when compared to similar studies and represents a range of demographic groups within regional and remote settings. It is the first study to apply the multi-domain CNAT-C scale in a Western sample and largely demonstrates validity of the factor structure for use in this setting. Finally, caregivers were supporting a very diverse range of cancer patients, including a variety of cancer types and treatment statuses. This enhances the diversity of the sample however future research may benefit from focusing on specific groups. There are some limitations worth noting. 
Selection bias is important to consider due to the recruitment process, since it required both cancer patients' nomination, and caregivers to agree to participate. Furthermore, given that these cancer patients were already utilizing the subsidized accommodation lodges, it is possible that their healthcare access and supportive care needs may be better fulfilled in comparison to the general remote cancer population.

\section{Conclusions}

Maintaining caregiver health, practical issues associated with travel, and emotional strain are all areas where regional and remote caregivers require more support. The age and gender of caregivers, along with time since diagnosis, and cancer type of the patient should be considered in determining the most appropriate supportive care for cancer caregivers.

Author Contributions: Conceptualization, J.D., S.K.C., J.F.A., S.M., B.C.G., M.I. and F.C.-W.; methodology, A.S. and B.C.G.; formal analysis, B.C.G. and A.S.; writing-original draft preparation, A.S. and B.C.G.; writing-review and editing, A.S., B.C.G., F.C.-W., S.M., M.I., J.F.A., J.D. and S.K.C.; supervision J.F.A., S.K.C. and J.D.; project administration, A.S., B.C.G. and F.C.-W. All authors have read and agreed to the published version of the manuscript.

Funding: This research received no external funding.

Institutional Review Board Statement: The study was conducted according to the guidelines of the Declaration of Helsinki, and approved by the Human Research Ethics Committee of the University of Southern Queensland (Ethics approval number: ref. H17REA152, approved 18 August 2017).

Informed Consent Statement: Informed consent was obtained from all subjects involved in the study.

Data Availability Statement: Data that support the findings of this study are available from the corresponding author upon reasonable request.

Acknowledgments: The authors would like to thank all the participants of this study. We would also like to acknowledge the invaluable support of our research volunteers, research assistants and Cancer Council lodge staff.

Conflicts of Interest: The authors declare no conflict of interest.

\section{Appendix A}

Table A1. Factor loadings for confirmatory factor analysis model 1 and 2.

\begin{tabular}{|c|c|c|}
\hline CNAT-C Item & Model 1 & Model 2 \\
\hline \multicolumn{3}{|l|}{ Health and psychological problems } \\
\hline My own health problems & 0.653 & 0.656 \\
\hline Concerns about the person I provide care for & 0.876 & 0.876 \\
\hline Depression & 0.883 & 0.882 \\
\hline Feelings of anger, irritability or nervousness & 0.947 & 0.946 \\
\hline Loneliness or feelings of isolation & 0.925 & 0.925 \\
\hline Feelings of vague anxiety & 0.933 & 0.934 \\
\hline \multicolumn{3}{|l|}{ Family/social support } \\
\hline Help with over-dependence from the person I am caring for & 0.878 & 0.878 \\
\hline Help with lack of appreciation of my caregiving from the person I care for & 0.852 & 0.850 \\
\hline Help with difficulties in family relationships after cancer diagnosis & 0.802 & 0.803 \\
\hline Help with difficulties in interpersonal relationships after cancer diagnosis & 0.874 & 0.876 \\
\hline Help with my own relaxation and my personal life & 0.964 & 0.963 \\
\hline \multicolumn{3}{|l|}{ Health-care staff } \\
\hline Being respected and treated as a person by my doctor & 0.940 & 0.940 \\
\hline Doctor to be clear, specific and honest in his/her explanation & 0.839 & 0.840 \\
\hline Seeing doctor quickly and easily when in need & 0.880 & 0.880 \\
\hline Being involved in the decision-making process in choosing any tests or treatments ... & 0.888 & 0.883 \\
\hline
\end{tabular}


Table A1. Cont.

\begin{tabular}{|c|c|c|}
\hline CNAT-C Item & Model 1 & Model 2 \\
\hline Cooperation and communication among health care staff & 0.860 & 0.857 \\
\hline Sincere interest and empathy from the nurses looking after the person I am caring for & 0.889 & 0.891 \\
\hline Nurses to explain treatment or care that is being given to the person I am caring for & 0.872 & 0.873 \\
\hline Nurses to promptly attend to the discomfort and pain of the person I am caring for & 0.794 & 0.796 \\
\hline \multicolumn{3}{|l|}{ Information } \\
\hline Information about the current status of the illness of the person I am caring for ... & 0.968 & 0.969 \\
\hline Information about tests and treatment & 0.950 & 0.951 \\
\hline Information about caring for the person with cancer (symptom management, diet, ... & 0.858 & 0.859 \\
\hline Guidelines or information about complementary and alternative medicine & 0.736 & 0.735 \\
\hline Information about hospitals or clinics and physicians who treat cancer & 0.739 & 0.742 \\
\hline Information about financial support for medical expenses, from government and/or ... & 0.853 & 0.853 \\
\hline Help with communication with the person I am caring for and/or friends and family ... & 0.805 & 0.779 \\
\hline Information about caregiving-related stress management & 0.934 & 0.931 \\
\hline \multicolumn{3}{|l|}{ Religious/spiritual support } \\
\hline Religious support & 0.124 & - \\
\hline Help in finding the meaning of my situation and coming to terms with it & 3.694 & - \\
\hline \multicolumn{3}{|l|}{ Hospital facilities and services } \\
\hline A designated hospital staff member who would be able to provide counselling for ... & 0.891 & 0.888 \\
\hline Guidance about hospital facilities and services & 0.918 & 0.913 \\
\hline Need for space reserved for caregivers & 0.810 & 0.813 \\
\hline A visiting nurse service for the home of the person I am caring for & 0.820 & 0.824 \\
\hline Opportunity to share experiences or information with other caregivers & 0.767 & 0.770 \\
\hline Welfare services (e.g., psychological counselling) for caregivers & 0.880 & 0.884 \\
\hline \multicolumn{3}{|l|}{ Practical support } \\
\hline Transportation service for getting to and from the hospital & 0.912 & 0.913 \\
\hline Treatment near home & 0.796 & 0.795 \\
\hline Lodging near hospital where the person I am caring for is treated & 0.743 & 0.743 \\
\hline Help with economic burden caused by cancer & 0.905 & 0.901 \\
\hline Someone to help me with housekeeping and/or childcare & 0.828 & 0.831 \\
\hline Assisted care in hospital or at the home of the person I am caring for & 0.920 & 0.922 \\
\hline
\end{tabular}

$$
\ldots=\text { item truncated. }
$$

Table A2. Confirmatory factor analysis fit statistics.

\begin{tabular}{ccc}
\hline & Model 1 & Model 2 \\
\hline Chi square & $155.88^{*}$ & $1090.819^{*}$ \\
RMSEA (95\% CI) & $0.066^{*}(0.061-0.071)$ & $0.050(0.044-0.055)$ \\
CFI/TLI & $0.915 / 0.908$ & $0.955 / 0.951$ \\
\hline$* p<0.01$. &
\end{tabular}

\section{References}

1. Australian Institute of Health and Welfare. Australian Burden of Disease Study: Impact and Causes of Illness and Death in Australia 2015; Australian Burden of Disease Series no. 19. Cat. no. BOD 22; AIHW: Canberra, Australia, 2019.

2. Goldsbury, D.E.; Yap, S.; Weber, M.F.; Veerman, L.; Rankin, N.; Banks, E.; Canfell, K.; O'Connell, D.L. Health services costs for cancer care in Australia: Estimates from the 45 and Up Study. PLoS ONE 2018, 13, e0201552. [CrossRef] [PubMed]

3. Girgis, A.; Lambert, S.; Lecathelinais, C. The supportive care needs survey for partners and caregivers of cancer survivors: Development and psychometric evaluation. Psycho-Oncology 2011, 20, 387-393. [CrossRef]

4. Yabroff, K.R.; Kim, Y. Time costs associated with informal caregiving for cancer survivors. Cancer 2009, 115, 4362-4373. [CrossRef]

5. Butow, P.N.; Price, M.A.; Bell, M.L.; Webb, P.M.; deFazio, A.; Friedlander, M. Caring for women with ovarian cancer in the last year of life: A longitudinal study of caregiver quality of life, distress and unmet needs. Gynecol. Oncol. 2014, 132, 690-697. [CrossRef]

6. Papastavrou, E.; Charalambous, A.; Tsangari, H. Exploring the other side of cancer care: The informal caregiver. Eur. J. Oncol. Nurs. 2009, 13, 128-136. [CrossRef] [PubMed] 
7. Sklenarova, H.; Krümpelmann, A.; Haun, M.W.; Friederich, H.C.; Huber, J.; Thomas, M.; Winkler, E.C.; Herzog, W.; Hartmann, M. When do we need to care about the caregiver? Supportive care needs, anxiety, and depression among informal caregivers of patients with cancer and cancer survivors. Cancer 2015, 121, 1513-1519. [CrossRef]

8. Girgis, A.; Lambert, S. Caregivers of cancer survivors: The state of the field. Cancer Forum 2009, 33, 167-171.

9. Stolz-Baskett, P.; Taylor, C.; Glaus, A.; Ream, E. Supporting older adults with chemotherapy treatment: A mixed methods exploration of cancer caregivers' experiences and outcomes. Eur. J. Oncol. Nurs. 2021, 50, 101877. [CrossRef]

10. Kim, Y.; Carver, C.S. Unmet needs of family cancer caregivers predict quality of life in long-term cancer survivorship. J. Cancer Surviv. 2019, 13, 749-758. [CrossRef]

11. Baudry, A.S.; Vanlemmens, L.; Anota, A.; Cortot, A.; Piessen, G.; Christophe, V. Profiles of caregivers most at risk of having unmet supportive care needs: Recommendations for healthcare professionals in oncology. Eur. J. Oncol. Nurs. 2019, 43, 101669. [CrossRef]

12. Lambert, S.D.; Harrison, J.D.; Smith, E.; Bonevski, B.; Carey, M.; Lawsin, C.; Paul, C.; Girgis, A. The unmet needs of partners and caregivers of adults diagnosed with cancer: A systematic review. BMJ Supportive Palliat. Care 2012, 2, 224-230. [CrossRef]

13. Heckel, L.; Fennell, K.M.; Reynolds, J.; Osborne, R.H.; Chirgwin, J.; Botti, M.; Ashley, D.M.; Livingston, P.M. Unmet needs and depression among carers of people newly diagnosed with cancer. Eur. J. Cancer 2015, 51, 2049-2057. [CrossRef] [PubMed]

14. Longstreth, M.; McKibbin, C.; Steinman, B.; Slosser Worth, A.; Carrico, C. Exploring Information and Referral Needs of Individuals with Dementias and Informal Caregivers in Rural and Remote Areas. Clin. Gerontol. 2020, 1-13. [CrossRef] [PubMed]

15. Lynagh, M.C.; Williamson, A.; Bradstock, K.; Campbell, S.; Carey, M.; Paul, C.; Tzelepis, F.; Sanson-Fisher, R. A national study of the unmet needs of support persons of haematological cancer survivors in rural and urban areas of Australia. Supportive Care Cancer 2018, 26, 1967-1977. [CrossRef] [PubMed]

16. Ugalde, A.; Blaschke, S.; Boltong, A.; Schofield, P.; Aranda, S.; Phipps-Nelson, J.; Chambers, S.K.; Krishnasamy, M.; Livingston, P.M. Understanding rural caregivers' experiences of cancer care when accessing metropolitan cancer services: A qualitative study. BMJ Open 2019, 9, e028315. [CrossRef] [PubMed]

17. Adashek, J.J.; Subbiah, I.M. Caring for the caregiver: A systematic review characterising the experience of caregivers of older adults with advanced cancers. ESMO Open 2020, 5, e000862. [CrossRef] [PubMed]

18. Williams, C.; Thorpe, R.; Harris, N.; Dickinson, H.; Barrett, C.; Rorison, F. Going home from hospital: The postdischarge experience of patients and carers in rural and remote Queensland. Aust. J. Rural. Health 2006, 14, 9-13. [CrossRef]

19. Goodwin, B.C.; Chambers, S.; Aitken, J.; Ralph, N.; March, S.; Ireland, M.; Rowe, A.; Crawford-Williams, F.; Zajdlewicz, L.; Dunn, J. Cancer-related help-seeking in cancer survivors living in regional and remote Australia. Psycho-Oncology 2021, 30, 1068-1076. [CrossRef]

20. Wang, T.; Molassiotis, A.; Chung, B.P.M.; Tan, J.Y. Unmet care needs of advanced cancer patients and their informal caregivers: A systematic review. BMC Palliat. Care 2018, 17, 96. [CrossRef]

21. Wu, V.S.; Smith, A.B.; Girgis, A. The unmet supportive care needs of Chinese patients and caregivers affected by cancer: A systematic review. Eur. J. Cancer Care 2020, e13269. [CrossRef]

22. Campbell, H.S.; Sanson-Fisher, R.; Taylor-Brown, J.; Hayward, L.; Wang, X.S.; Turner, D. The cancer support person's unmet needs survey: Psychometric properties. Cancer 2009, 115, 3351-3359. [CrossRef] [PubMed]

23. Hodgkinson, K.; Butow, P.; Hunt, G.E.; Pendlebury, S.; Hobbs, K.M.; Lo, S.K.; Wain, G. The development and evaluation of a measure to assess cancer survivors' unmet supportive care needs: The CaSUN (Cancer Survivors' Unmet Needs measure). Psycho-Oncology 2007, 16, 796-804. [CrossRef]

24. Shin, D.W.; Park, J.H.; Shim, E.J.; Park, J.H.; Choi, J.Y.; Kim, S.G.; Park, E.C. The development of a comprehensive needs assessment tool for cancer-caregivers in patient-caregiver dyads. Psycho-Oncology 2011, 20, 1342-1352. [CrossRef]

25. Kim, H.; Yi, M. Unmet needs and quality of life of family caregivers of cancer patients in South Korea. Asia Pac. J. Oncol. Nurs. 2015, 2, 152-159. [CrossRef] [PubMed]

26. Muthén, L.K.; Muthén, B.O. Mplus: Statistical Analysis with Latent Variables: User's Guide; Version 8; Muthén \& Muthén: Los Angeles, CA, USA, 2017.

27. Morrison, V.; Henderson, B.J.; Zinovieff, F.; Davies, G.; Cartmell, R.; Hall, A.; Gollins, S. Common, important, and unmet needs of cancer outpatients. Eur. J. Oncol. Nurs. 2012, 16, 115-123. [CrossRef] [PubMed]

28. Soothill, K.; Morris, S.M.; Harman, J.; Francis, B.; Thomas, C.; McIllmurray, M.B. The significant unmet needs of cancer patients: Probing psychosocial concerns. Supportive Care Cancer 2001, 9, 597-605. [CrossRef]

29. Kim, Y.; Kashy, D.A.; Spillers, R.L.; Evans, T.V. Needs assessment of family caregivers of cancer survivors: Three cohorts comparison. Psycho-Oncology 2010, 19, 573-582. [CrossRef] [PubMed]

30. Soothill, K.; Morris, S.M.; Harman, J.C.; Francis, B.; Thomas, C.; McIllmurray, M.B. Informal carers of cancer patients: What are their unmet psychosocial needs? Health Soc. Care Community 2001, 9, 464-475. [CrossRef]

31. Gunn, K.M.; Olver, I.; Skrabal Ross, X.; Harrison, N.; Livingston, P.M.; Wilson, C. Improving Survivors' Quality of Life PostTreatment: The Perspectives of Rural Australian Cancer Survivors and Their Carers. Cancers 2021, 13, 1600. [CrossRef] [PubMed]

32. Perz, J.; Ussher, J.M.; Butow, P.; Wain, G. Gender differences in cancer carer psychological distress: An analysis of moderators and mediators. Eur. J. Cancer Care 2011, 20, 610-619. [CrossRef]

33. Ussher, J.M.; Sandoval, M. Gender differences in the construction and experience of cancer care: The consequences of the gendered positioning of carers. Psychol. Health 2008, 23, 945-963. [CrossRef] 
34. Hagedoorn, M.; Sanderman, R.; Bolks, H.N.; Tuinstra, J.; Coyne, J.C. Distress in couples coping with cancer: A meta-analysis and critical review of role and gender effects. Psychol. Bull. 2008, 134, 1-30. [CrossRef] [PubMed]

35. Kim, Y.; Mitchell, H.R.; Ting, A. Application of psychological theories on the role of gender in caregiving to psycho-oncology research. Psycho-Oncology 2019, 28, 228-254. [CrossRef]

36. Ochoa, C.Y.; Buchanan Lunsford, N.; Lee Smith, J. Impact of informal cancer caregiving across the cancer experience: A systematic literature review of quality of life. Palliat. Supportive Care 2020, 18, 220-240. [CrossRef]

37. Goerling, U.; Bergelt, C.; Müller, V.; Mehnert-Theuerkauf, A. Psychosocial Distress in Women With Breast Cancer and Their Partners and Its Impact on Supportive Care Needs in Partners. Front. Psychol. 2020, 11, 564079. [CrossRef] [PubMed]

38. Kim, Y.; Loscalzo, M.J.; Wellisch, D.K.; Spillers, R.L. Gender differences in caregiving stress among caregivers of cancer survivors. Psycho-Oncology 2006, 15, 1086-1092. [CrossRef] [PubMed]

39. Spatuzzi, R.; Giulietti, M.V.; Romito, F.; Reggiardo, G.; Genovese, C.; Passarella, M.; Raucci, L.; Ricciuti, M.; Merico, F.; Rosati, G.; et al. Becoming an older caregiver: A study of gender differences in family caregiving at the end of life. Palliat. Supportive Care 2021, 1-7. [CrossRef]

40. Maxwell, A.; Özmen, M.; Iezzi, A.; Richardson, J. Deriving population norms for the AQoL-6D and AQoL-8D multi-attribute utility instruments from web-based data. Qual. Life Res. 2016, 25, 3209-3219. [CrossRef] [PubMed]

41. Kim, Y.; Kashy, D.A.; Evans, T.V. Age and attachment style impact stress and depressive symptoms among caregivers: A prospective investigation. J. Cancer Surviv. 2007, 1, 35-43. [CrossRef]

42. Wang, T.; Mazanec, S.R.; Voss, J.G. Needs of Informal Caregivers of Patients With Head and Neck Cancer: A Systematic Review. Oncol. Nurs. Forum 2021, 48, 11-29. [CrossRef]

43. Bergerot, C.D.; Bergerot, P.G.; Philip, E.J.; De Domenico, E.B.L.; Manhaes, M.F.M.; Pedras, R.N.; Salgia, M.M.; Dizman, N.; Ashing, K.T.; Li, M.; et al. Assessment of distress and quality of life in rare cancers. Psycho-Oncology 2018, 27, 2740-2746. [CrossRef] [PubMed]

44. Horick, N.K.; Manful, A.; Lowery, J.; Domchek, S.; Moorman, P.; Griffin, C.; Visvanathan, K.; Isaacs, C.; Kinney, A.Y.; Finkelstein, D.M. Physical and psychological health in rare cancer survivors. J. Cancer Surviv. 2017, 11, 158-165. [CrossRef]

45. Applebaum, A.J.; Polacek, L.C.; Walsh, L.; Reiner, A.S.; Lynch, K.; Benvengo, S.; Buthorn, J.; Atkinson, T.M.; Mao, J.J.; Panageas, K.S.; et al. The unique burden of rare cancer caregiving: Caregivers of patients with Erdheim-Chester disease. Leuk. Lymphoma 2020, 61, 1406-1417. [CrossRef] [PubMed]

46. Taylor, J.; Fradgley, E.; Clinton-McHarg, T.; Byrnes, E.; Paul, C. Access to support for Australian cancer caregivers: In-depth qualitative interviews exploring barriers and preferences for support. J. Psychosoc. Oncol. Res. Pract. 2021, 3, e047. [CrossRef]

47. Alfano, C.M.; Jefford, M.; Maher, J.; Birken, S.A.; Mayer, D.K. Building Personalized Cancer Follow-up Care Pathways in the United States: Lessons Learned From Implementation in England, Northern Ireland, and Australia. Am. Soc. Clin. Oncol. Educ. Book 2019, 39, 625-639. [CrossRef]

48. Watson, E.K.; Rose, P.W.; Neal, R.D.; Hulbert-Williams, N.; Donnelly, P.; Hubbard, G.; Elliott, J.; Campbell, C.; Weller, D.; Wilkinson, C. Personalised cancer follow-up: Risk stratification, needs assessment or both? Br. J. Cancer 2012, 106, 1-5. [CrossRef]

49. Sabo, K.; Chin, E. Self-care needs and practices for the older adult caregiver: An integrative review. Geriatr. Nurs. 2021, 42, 570-581. [CrossRef] 